\title{
Myoclonus in a Patient with Acute Kidney Injury: A Rare Presentation of Gabapentin Toxicity
}

\author{
Muzammil Khan", Shaza Khalid, Asghar Marwat, Salah Aldergash, Medha Joshi, Varun Malhotra \\ Department of Internal Medicine, Conemaugh Memorial Medical Center/ Temple University, Johnstown, PA, USA \\ *Corresponding author
}

\begin{abstract}
Gabapentin, an anti-epileptic drug (AED) is commonly used off label for management of neuropathic pain and psychiatric disorders. Dosing of gabapentin requires taking into consideration the renal function as it is entirely cleared by the kidneys. Acute kidney injury and end stage renal disease increase the risk of developing myoclonic activity, an infrequent manifestation of gabapentin toxicity. We report a case of confusion and myoclonic activity related to gabapentin toxicity coincident with acute kidney injury that resolved with discontinuation of gabapentin and treatment with intravenous fluid hydration. As gabapentin is commonly used off label across multiple specialties, clinician recognition of the significance of renal dosing and understanding of the potential association with myoclonus and neurotoxicity is important.
\end{abstract}

Keywords: gabapentin toxicity

Cite This Article: Muzammil Khan, Shaza Khalid, Asghar Marwat, Salah Aldergash, Medha Joshi, and Varun Malhotra, "Myoclonus in a Patient with Acute Kidney Injury: A Rare Presentation of Gabapentin Toxicity." American Journal of Medical Case Reports, vol. 5, no. 8 (2017): 232-233. doi: 10.12691/ajmcr-5-8-8.

\section{Introduction}

Gabapentin is a widely used medicine in clinical practice to treat neuropathy and musculoskeletal pain [1]. Gabapentin dosing depends on the kidney function [3,4]. Serious neurological toxicity may result in patients with impaired kidney function.

\section{Case}

A 55 year old female with history of bipolar disorder, depression, hypertension, and chronic back pain presented with uncontrolled movements of all four extremities and increased propensity to fall for 1 week prior to admission. The patient had no history of renal disease. Her medications on admission included acetaminophen, clonazepam, gabapentin 1500 mg/day, hydrocodone, lisinopril, hydrochlorothiazide, quetiapine, omeprazole, sumatriptan, and simvastatin. Physical examination did not reveal any focal neurological deficit but revealed a combination of myoclonus and asterixis with unbalanced gait; clinically the patient appeared to be volume depleted with dry mucous membranes. Abnormal lab results on admission included sodium level of $132 \mathrm{mmol} / \mathrm{L}$, blood urea nitrogen (BUN) $73 \mathrm{mg} / \mathrm{dL}$, creatinine (Cr) $3.7 \mathrm{mg} / \mathrm{dL}$ as compared to BUN $17 \mathrm{mg} / \mathrm{dL}$ and $\mathrm{Cr} 0.7 \mathrm{mg} / \mathrm{dL}$ at baseline 8 weeks ago, and white blood cell count $1.34 \times 10^{3} / \mathrm{uL}$. Urinalysis revealed 2+ white blood cell, nitrite positive and a serum gabapentin level $35.4 \mathrm{ug} / \mathrm{mL}$, normal serum gabapentin reference ranges from 4.0 - $16.0 \mathrm{ug} / \mathrm{mL}$. CT scan of head without contrast was normal, EEG showed generalized mild-moderate slowing and generalized discharges with triphasic morphology, consistent with a diffuse disturbance of cerebral function or encephalopathy secondary to metabolic, toxic, inflammatory, or other systemic processes. No epileptiform discharges or seizures were identified on the recordings. Treatment with IV hydration (IVF) and discontinuation of gabapentin resulted in significant improvement in all of her symptoms within 24 hours. Patient was discharged with normal renal function, BUN $20 \mathrm{mg} / \mathrm{dL}$, creatinine $0.7 \mathrm{mg} / \mathrm{dL}$ and resolved myoclonus. Gabapentin was held on discharge.

\section{Discussion}

Gabapentin is a commonly used AED with analgesic effects that makes it a useful drug for neuropathic pain ${ }^{[1]}$. Gabapentin is used more often for neuropathic pain than as an AED [2]. Gabapentin is well absorbed from the gastrointestinal tract with bioavailability reaching 60\% [3]. The concentration of gabapentin in the brain is usually higher than the plasma gabapentin concentration as it readily crosses the blood brain barrier (BBB), as well as actively being transported across the BBB $[3,4]$. Excretion of gabapentin depends entirely on renal function as it is neither protein bound in circulation nor metabolized in the body. Its concentration in the body rises as the creatinine clearance falls [3,4], which makes renal dose adjustments necessary to avoid toxicity. The half-life of gabapentin has been estimated to be 132 hours in patients with end stage renal disease or on non-hemodialysis days as compared with 5 to 7 hours in patients with normal renal function [5], thus emphasizing the importance of renal dose adjustments of 
gabapentin. Our patient was receiving gabapentin 1500 $\mathrm{mg}$ /day with a baseline estimated glomerular filtration rate (eGFR) of $87 \mathrm{~mL} / \mathrm{min}$, a dose appropriate for normal renal function, but upon presentation to hospital, patient's eGFR was $13 \mathrm{~mL} / \mathrm{min}$.

Gabapentin is usually well-tolerated if dose adjusted for renal function; however, several published case reports have described adverse events/effects of gabapentin that include myoclonus, myopathy, neutropenia, hypoglycemia, changes in mental status, drowsiness and coma, occurring more commonly in patients with renal dysfunction [6,7]. Consistent with the symptoms described in these case reports, our patient presented with confusion, increased propensity to fall and myoclonus. In cases of symptomatic toxicity, dialysis is used to rapidly clear the serum of gabapentin. Both intermittent and continuous renal replacement therapy have shown efficacy in treating gabapentin induced myoclonus and neurotoxicity $[7,8]$. In contrast, our patient's renal function, myoclonus and mental status improved with IVF, and therefore did not receive any dialysis.

On presentation, our patient had a serum gabapentin level of $35 \mathrm{ug} / \mathrm{mL}$, repeat gabapentin levels were not obtained as the patient was improving clinically. Of note, serum concentrations greater than $15 \mathrm{ug} / \mathrm{mL}$ have been shown to be associated with symptomatic toxicity [9].

\section{Conclusion}

1. It is important to educate physicians from various specialties regarding the adverse effects of gabapentin and the necessity of renal dosing as gabapentin is commonly used off label for various indications across multiple specialties.

2. Gabapentin requires renal dosing in patients with chronic kidney disease and those at risk of acute kidney injury. We recommend holding gabapentin while acute illness or dehydration is present, unless used as AED, in which case renal function should be monitored closely.

3. Symptomatic gabapentin toxicity in patients with acute kidney injury requires consultation with a neurologist and nephrologist for appropriate management.

\section{References}

[1] Backonja M, Beydoun A, Edwards KR, Schwartz SL, Fonseca V, Hes M, LaMoreaux L, Garofalo E, Gabapentin Diabetic Neuropathy Study Group. Gabapentin for the symptomatic treatment of painful neuropathy in patients with diabetes mellitus: a randomized controlled trial. JAMA. 1998 Dec 2; 280(21): 1831-6.

[2] Mack A. Examination of the evidence for off-label use of gabapentin. Journal of Managed Care Pharmacy. 2003 Nov; 9(6): 559-68.

[3] Blum RA, Comstock TJ, Sica DA, Schultz RW, Keller E, Reetze P, Bockbrader H, Tuerck D, Busch JA, Reece PA, Sedman AJ. Pharmacokinetics of gabapentin in subjects with various degrees of renal function. Clinical Pharmacology \& Therapeutics. 1994 Aug 1; 56(2): 154-9.

[4] Catterall WA. Structure and regulation of voltage-gated Ca2+ channels. Annual Review of Cell and Developmental Biology. 2000 Nov; 16(1): 521-55.

[5] Wong MO, Eldon MA, Keane WF, Türck D, Bockbrader HN, Underwood BA, Sedman AJ, Halstenson CE. Disposition of gabapentin in anuric subjects on hemodialysis. The Journal of Clinical Pharmacology. 1995 Jun 1; 35(6): 622-6.

[6] Miller A, Price G. Gabapentin toxicity in renal failure: the importance of dose adjustment. Pain Medicine. 2009 Jan 1; 10(1): 190-2.

[7] Hung TY, Seow VK, Chong CF, Wang TL, Chen CC. Gabapentin toxicity: an important cause of altered consciousness in patients with uraemia. BMJ Case Reports. 2009 Jan 1; 2009: bcr1120081268.

[8] Guddati AK, Zafar Z, Cheng JT, Mohan S. Treatment of gabapentin-induced myoclonus with continuous renal replacement therapy. Indian Journal of Nephrology. 2012 Jan 1; 22(1): 59.

[9] Zand L, McKian KP, Qian Q. Gabapentin toxicity in patients with chronic kidney disease: a preventable cause of morbidity. The American Journal of Medicine. 2010 Apr 30; 123(4): 367-73. 